\title{
Avaliação do Nível de Maturidade em Teste de Software em Micro e Pequenas Empresas do Estado de Goiás
}

\author{
Alternative Title: Maturity Level Assessment in Software Testing in Small and \\ Medium-Sized Enterprises of the State of Goiás
}

\author{
Adailton F. Araújo \\ Instituto de Informática \\ Universidade Federal de \\ Goiás (UFG) \\ Goiânia, Brasil \\ adailton@inf.ufg.br
}

\author{
Cássio L. Rodrigues \\ Instituto de Informática \\ Universidade Federal de \\ Goiás (UFG) \\ Goiânia, Brasil \\ cassio@inf.ufg.br \\ Celso G. Camilo \\ Instituto de Informática \\ Universidade Federal de \\ Goiás (UFG) \\ Goiânia, Brasil \\ celso@inf.ufg.br
}

\author{
Auri M.R. Vincenzi \\ Instituto de Informática \\ Universidade Federal de \\ Goiás (UFG) \\ Goiânia, Brasil \\ auri@inf.ufg.br
}

\begin{abstract}
RESUMO
O teste de software é um importante componente que leva a produção de software com qualidade. Este artigo apresenta os resultados da aplicação de um arcabouço para avaliação do nível de maturidade em Teste de Software no contexto de Micro e Pequenas Empresas (MPEs) com base no modelo TMMi. O arcabouço é composto por um questionário de avaliação construído com base nas subpráticas do modelo TMMi, material de apoio com exemplos de artefatos requeridos para garantir o atendimento a cada questão do questionário e um suporte automatizado para apoiar a aplicação do questionário, visando a autoavaliação de MPEs. O arcabouço foi aplicado em dez empresas, diante dos resultados apresentados, pode-se concluir que a maturidade das empresas em teste de software é baixa e que as empresas avaliaram positivamente a adequação do arcabouço desenvolvido para o contexto de MPEs.
\end{abstract}

\section{Palavras-Chave}

Avaliação da Maturidade de Processo de Teste, Melhoria de Processo de Teste de Software, TMMi, TMM

\begin{abstract}
Software testing is an important component that leads to quality software production. This paper presents the results of a framework for assessing the level of maturity in
\end{abstract}

Permission to make digital or hard copies of all or part of this work for personal or classroom use is granted without fee provided that copies are not made or distributed for profit or commercial advantage and that copies bear this notice and the full citation on the first page. To copy otherwise, to republish, to post on servers or to redistribute to lists, requires prior specific permission and/or a fee.

SBSI 2015, May 26th-29th, 2015, Goiânia, Goiás, Brazil

Copyright SBC 2015.
Software Testing application in the context of Small and Medium-Sized Enterprises (SMEs) based on TMMi model. Our framework includes an evaluation questionnaire based on TMMi sub-practices, support tools with examples of artifacts required to ensure that the questionnaire is thoroughly completed, as well as an automated tool support for its application, enabling SMEs to carry out self-assessment. The framework was applied in ten companies and before the results presented, it can be concluded that the companies maturity in software testing is low and that the companies positively assessed the adequacy of the framework developed for the context of SMEs.

\section{Categories and Subject Descriptors}

D.2.9 [Software Engineering]: Software process modelsMaturity Assessment of Test Process

\section{General Terms}

Software Quality, Management, Standardization.

\section{Keywords}

Maturity Assessment of Test Process, Software Test Process Improvement, TMMi, TMM.

\section{INTRODUÇÃO}

As Micro e Pequenas Empresas (MPEs) de software são uma peça muito importante na engrenagem da economia mundial e representam 99,2\% das empresas de software do mundo [1]. Em 2013, a participação de programas de computador desenvolvidos no Brasil atingiu $21.4 \%$ do total do mercado brasileiro de software. Este mercado é explorado por 11.232 empresas, dedicadas ao desenvolvimento, produção e distribuição de software e de prestação de serviços. Daquelas que atuam no desenvolvimento e produção de software (2.708), 94\% são classificadas como MPEs [5]. Além disso, 
as empresas de desenvolvimento de software com menos de 10 empregados representam 93\% das empresas da Europa e $56 \%$ nos EUA [8]. Assim, a grande maioria das empresas de desenvolvimento de software do mundo são classificadas como MPEs [7], o que caracteriza a importância das MPEs para este mercado.

Produzir software de qualidade é um desafio para MPEs. Em geral, as MPEs possuem formas limitadas de serem reconhecidas como empresas desenvolvedoras de produtos de software de qualidade [8]. O mercado de software está cada vez mais exigente e as MPEs necessitam melhorar continuamente a qualidade de seus produtos para se adequarem às exigências do mercado.

A qualidade de um sistema ou produto é amplamente influenciada pela qualidade do processo utilizado, e requer o fornecimento de uma base para maximizar a produtividade das pessoas e o uso da tecnologia para se tornar mais competitivo no mercado [11]. Um bom processo sozinho não ajuda a organização a desenvolver produtos de sucesso, mas um bom processo é necessário para a construção de um produto de sucesso [8].

Percebendo a importância da utilização de processos, a indústria de software busca certificações em diversos modelos que enfatizam a disciplina no uso de processos, tais como certificações CMMI (Capability Maturity Model Integration) [11] e MPS.BR (Melhoria do Processo de Software Brasileiro) [9]. Há um custo mínimo para iniciar um programa de melhoria de processo de software, independentemente do tamanho da empresa e, assim, esse investimento, que comparado ao faturamento de uma grande empresa pode ser irrelevante, se torna inviável para uma MPE [3]. Além disso, a melhoria de processo de software em pequenas empresas requer uma atenção especial quanto à aplicação de modelos e padrões que foram projetados a partir do ponto de vista de grandes organizações [17].

O teste é um importante componente no ciclo de vida de desenvolvimento que leva a produção de software com maior qualidade [15]. O problema de modelos de maturidade como CMMI e MPS.BR é que, além de não darem a devida importância para o teste, a exigência do processo de teste só é feita em seus níveis de maturidade mais elevados, níveis esses que geralmente não são alcançados pelas MPEs. No MPS.BR, que é voltado para as MPEs, somente $8 \%$ das empresas certificadas possuem certificação nos níveis mais elevados A, B, C e D [13], que exigem o processo de Verificação e Validação.

A indústria de software tem focado na melhoria de processo de teste para aumentar seu desempenho [15]. Com isso, surgiram modelos de maturidade de teste de software como TMM ( Test Maturity Model) [4] e TMMi (Test Maturity Model Integration) [16], com foco na melhoria de processo de teste. Porém, os altos custos que envolvem essas certificações e o fato desses modelos serem projetados a partir do ponto de vista de grandes organizações, as tornam inacessíveis para as MPEs. O modelo TMM é um dos modelos de maturidade de teste mais empregado mundialmente e o TMMi é uma evolução do TMM [4]. Entretanto, até o presente momento, ao contrário do TMM, não existe um questionário de assessment disponível gratuitamente e que possa ser utilizado por MPEs para a realização de uma autoavaliação.

No trabalho [2], os autores definiram um arcabouço para avaliação do nível de maturidade do processo de teste baseado nas práticas do TMMi, que fosse adequado à realidade de MPEs. Devido às restrições financeiras as MPEs necessitam de um método que as permitam realizar uma autoavaliação do seu nível de maturidade no processo de teste, pois os investimentos requeridos por uma avaliação formal não são compatíveis com a realidade das MPEs. Adicionalmente, para realizar uma autoavaliação, as MPEs precisam lidar com a baixa maturidade em processo de teste e em melhoria de processo. O referido trabalho lida com estes problemas criando um material de apoio para que as empresas possam se autoavaliar, sem possuir um conhecimento avançado do modelo. O cerne deste material de apoio é um questionário objetivo para avaliação da maturidade do processo de teste segundo o modelo TMMi. Para cada questão, são apresentados exemplos de como a empresa pode atender as exigências do modelo. Além disso, um suporte automatizado para facilitar a realização da avaliação e consolidação dos resultados foi desenvolvido. Este suporte pode reduzir o esforço de avaliação porque considera as dependências entre as questões para ajudar a reduzir o número de questões que precisam ser respondidas.

Neste trabalho, apresentamos os resultados da aplicação e validação do arcabouço em micro e pequena empresas do Estado de Goiás. O restante deste trabalho está organizado da seguinte forma. Seção 2 apresenta os trabalhos relacionados. Seção 3 descreve o arcabouço. Seção 4 descreve o estudo de caso realizado, explicando o contexto em que o arcabouço foi aplicado. Seção 5 apresenta os resultados da aplicação e avaliação do arcabouço. Por fim, a conclusão é apresentada na Seção 6 .

\section{JUSTIFICATIVAS PARA A ESCOLHA DO ARCABOUÇO}

Diversos trabalhos e ferramentas já foram desenvolvidos com o objetivo de apoiar a avaliação do nível de maturidade de processos de desenvolvimento e de teste. O trabalho de Tayamanon et al. [15] propõe uma ferramenta de suporte baseado no TMM-AM para que cada organização possa avaliar o seu processo de teste por si só. O primeiro passo para construção da ferramenta foi analisar cada questão do questionário TMM-AM e levantar os produtos de trabalho, baseados nas normas IEEE 730 e 829, necessários para atender a cada questão. A avaliação pela ferramenta é feita por meio da comparação de cada produto de trabalho padrão com o produto de trabalho produzido pela organização. O problema na aplicação dessa abordagem em MPEs é que, conforme levantado por $\mathrm{Ng}$ et al. [10], a maioria das MPEs não implementam os padrões de documentação e acabam criando documentos customizados. A frequência com que ocorreria a conformidade parcial da documentação produzida pelas empresas, fato que não foi tratado na ferramenta, prejudicaria uma avaliação utilizando essa ferramenta no contexto de MPEs. O tratamento dessa situação se remeteria novamente para uma avaliação subjetiva do nível de atendimento de cada artefato, cenário não adequado para MPEs.

A Appraisal Assistant é uma ferramenta de avaliação que foi desenvolvida pelo Software Quality Institute - SQI, na Universidade de Griffith [14]. Esta ferramenta da suporte para avaliação da capacidade ou maturidade do processo de uma organização à partir de modelos de avaliação como SCAMPI (CMMI) e ISO/IEC 15504. A ferramenta utiliza múltiplos modelos de referência, incluindo CMMI V1.1, V1.2 e V1.3, ISO/IEC 12207, e outros. Foram realizados alguns 
testes com a ferramenta, que mostrou-se de fácil usabilidade. Entretanto, essa é uma ferramenta para avaliação da maturidade do processo de desenvolvimento e não é focada em avaliação de processo de teste.

No trabalho de Höhn [6] foi desenvolvido um arcabouço com o objetivo de agregar conhecimentos gerais na área de teste e disponibiliza-lo para a comunidade com a intenção de facilitar a sua transferência, a definição e a melhoria de processos de teste, com mais qualidade. A ferramenta KITTool disponibiliza dois tipos de diagnósticos do processo de teste, um com base nos objetivos do TMMi e outro com base em suas práticas. Para a abordagem baseada nos objetivos são apresentadas as áreas de processo e os seus objetivos, o avaliador deve fornecer notas $(0,4,6,8$ e 10) para cada objetivo que representem o seu processo de teste. A segunda abordagem, baseada nas práticas, utiliza o método de avaliação SCAMPI do CMMI, no qual são apresentados os objetivos e as práticas relacionadas a cada objetivo e o avaliador deve atribuir notas $(0,25 \%, 50 \%, 75 \%$ e $100 \%)$ às práticas, baseando-se em uma tabela de referência, que representa o quanto da prática já foi implantada no processo em avaliação. Porém a aplicação da ferramenta exige um alto nível de conhecimento do modelo TMMi e não possui foco voltado para as MPEs.

O modelo de Melhoria de Processo de Teste de Software Brasileiro (MPT.Br)[12] é referência para definição, implantação e melhoria de processo de teste para pequenas empresas. Entretanto, não disponibiliza uma ferramenta pública que possa ser utilizada de maneira objetiva na avaliação de processos de teste.

Em relação aos trabalhos discutidos anteriormente, os diferenciais do arcabouço proposto em [2] são os seguintes:

- O arcabouço disponibiliza material de apoio para auxiliar uma MPE na realização de uma autoavaliação da sua maturidade em processo de teste;

- O suporte automatizado permite o vínculo das evidências utilizadas para comprovar o atendimento a cada objetivo/prática do TMMi. Isto permite uma visibilidade de como a empresa está atendendo ao modelo, facilitando a reavaliação do processo e possíveis auditorias externas;

- O suporte automatizado disponibiliza a relação de dependência entre as perguntas do questionário de avaliação, podendo reduzir a quantidade de itens a serem verificados ou até mesmo indicar inconsistências no processo de avaliação; e

- O arcabouço foi aplicado e avaliado por meio de um estudo da caso.

\section{O ARCABOUÇO}

Nesta seção será apresentado o arcabouço de avaliação do nível de maturidade em Teste de Software segundo o modelo TMMi criado em [2].

\subsection{Questionário de Avaliação}

O modelo TMMi possui um conjunto de cinco níveis de maturidade, cada nível de maturidade possui um conjunto de áreas de processo, cada área de processo possui um conjunto de metas, cada meta possui um conjunto de práticas e cada prática possui um conjunto de subpráticas. A subprática é uma descrição detalhada que fornece orientação para a interpretação e implementação de uma prática específica [16]. Por exemplo, na primeira coluna da Tabela 1 são apresentadas parte das subpráticas referentes a prática Definir Objetivos de Teste, da meta Estabelecer Políticas de Teste, da área de processo Estratégias de Políticas e Testes, relacionada ao Nível 2 de maturidade.

Tabela 1: Algumas das questões definidas com base nas subpráticas do TMMi

\begin{tabular}{|l|l|}
\hline Subprática & Questão \\
\hline $\begin{array}{l}\text { 1. Estudar as necessidades e objetivos de } \\
\text { negócios; }\end{array}$ & OR \\
\hline $\begin{array}{l}\text { 2. Permitir a interposição de comentá- } \\
\text { rios para esclarecer as necessidades e ob- } \\
\text { jetivos de negócios, caso seja necessário; }\end{array}$ & OR \\
\hline $\begin{array}{l}\text { 3. Definir objetivos de teste rastreáveis } \\
\text { às necessidades e objetivos de negócios; }\end{array}$ & $\begin{array}{l}\text { (3) Existem objetivos de } \\
\text { teste definidos com base } \\
\text { nas necessidades e objeti- } \\
\text { vos de negócio? }\end{array}$ \\
\hline $\begin{array}{l}\text { 4. Revisar os objetivos de teste com as } \\
\text { partes interessadas; }\end{array}$ & OR \\
\hline $\begin{array}{l}5 . \text { Reavaliar os objetivos de teste con- } \\
\text { forme o caso, por exemplo, anualmente }\end{array}$ & $\begin{array}{l}\text { (5) Os objetivos de teste } \\
\text { são revisados periodica- } \\
\text { mente? }\end{array}$ \\
\hline
\end{tabular}

Essa é a estrutura de todas áreas de processo relacionadas aos Níveis 2,3 e 4 . As áreas de processo relacionadas ao Nível 5 , não possuem práticas nem subpráticas e as práticas genéricas, que são aplicáveis a todas as áreas de processo, não possuem subpráticas. O trabalho desenvolvido por Höhn [6] disponibilizou duas formas de avaliação, uma com base nas metas e outra com base nas práticas do modelo. Ao avaliar o modelo TMMi constatou-se que para uma autoavaliação no contexto das MPEs os detalhes das subpráticas seriam fundamentais, já que neste caso o avaliador não possui conhecimento dos detalhes requeridos para o atendimento de uma meta/prática. Diante disso foi construído um questionário para avaliar a aderência ao TMMi com base nas suas subpráticas.

O questionário foi criado, considerando os seguintes critérios:

- Foram criadas questões apenas para as subpráticas que representam um resultado a ser atendido pelo modelo, as demais subpráticas que se tratam de orientações para implementação da prática não tiveram questões relacionadas. A Tabela 1 apresenta exemplos de questões criadas com base em subpráticas nas subpráticas 3 e 5. Para as subpráticas sem questões relacionadas (1, 2 e 4) foi atribuída a sigla OR (Orientação);

- Foi mantida a rastreabilidade entre as questões e as subpráticas do modelo para facilitar futuros refinamentos e manutenções das questões. Conforme pode ser visto na Tabela 1 , os números entre parênteses ao lado de cada questão demonstra essa rastreabilidade;

- Para os casos nos quais o modelo não disponibiliza subpráticas (todas as práticas genéricas e algumas específicas pontuais), foi elaborada uma questão com base na descrição da prática. Por exemplo, para a prática Distribuir as Políticas para os Interessados, que possui a descrição "As políticas e os objetivos do teste são apresentados e explicados às partes interessadas que estão envolvidas ou não com a atividade de teste", foi elaborada a seguinte pergunta: As políticas de teste foram 
apresentadas aos interessados (que estão envolvidos ou não com o teste)?; e

- Para os casos nos quais o modelo não disponibiliza práticas (áreas de processo relacionadas ao nível 5), foi elaborada uma questão com base na descrição da meta. Por exemplo, para a meta Selecionar Melhorias de Processo de Teste, que possui a descrição "São escolhidas melhorias de processo de teste que contribuam para atingir a qualidade do produto e objetivos de desempenho do processo", foi elaborada a seguinte pergunta: São selecionadas melhorias de processo de teste que contribuam para atingir a qualidade do produto e objetivos desempenho do processo?

Como resultado foi elaborado um questionário com 261 questões, para cobrir todas as áreas de processo do modelo TMMi. A definição do nível de maturidade, a partir de respostas atribuídas para o questionário, é realizada conforme o método de avaliação previsto no modelo TMM [4], o qual prevê como respostas para cada pergunta as alternativas: Sim, Não, Não se Aplica e Não Conhecido.

\subsection{Material de Apoio}

Para possibilitar uma autoavaliação por parte das MPEs, sem que o avaliador possua um conhecimento avançado do modelo TMMi, para cada questão são apresentados exemplos de artefatos típicos de trabalhos frequentemente utilizados para comprovar que a organização atende ao resultado esperado pelo modelo. O modelo TMMi já disponibiliza exemplos para muitas de suas subpráticas. Diante disso os exemplos foram definidos considerando os seguintes critérios:

- Para as questões relacionadas as práticas as quais o TMMi disponibiliza exemplos, foram utilizados os exemplos do próprio modelo. Alguns desses exemplos são extensos, entretanto, com o objetivo de impedir que o material de apoio se torne um gargalo na avaliação, foi estabelecido um limite de no máximo 5 exemplos por item exemplificado. A seleção dos exemplos foi feita com a leitura de todos os exemplos, procurou-se selecionar os que mais claramente estavam relacionados com o item exemplificado;

- Para as questões relacionadas as práticas que não possuía exemplos no TMMi, procurou-se extrair os exemplos das demais práticas que demonstravam detalhes de implementação do modelo; e

- Para as questões que não se encaixavam em nenhum dos itens anteriores foi utilizada a experiência e/ou outras referências na proposição do artefato.

Como resultados foram produzidos exemplos para as 261 questões do questionário. A Tabela 2 mostra alguns dos exemplos definidos. As questões (3) e (5) foram derivadas da Tabela 1 e as demais de outras subpráticas do TMMi. Para a questão (1) "Foram selecionadas técnicas de projeto de teste que fornecem uma ade-quada cobertura de teste sobre riscos de produtos definidos?" os seguintes exemplos foram indicados: Partição de Equivalência, Análise do Valor Limite, Tabela de Decisão, Teste de Transição de Estados e Teste de Caso de Uso.
Tabela 2: Alguns dos exemplos definidos com base no modelo TMMi e experiência

\begin{tabular}{|c|c|}
\hline Questão & Material de Apoio \\
\hline $\begin{array}{l}\text { (1)Foram selecionadas } \\
\text { técnicas de projeto de } \\
\text { teste que fornecem uma } \\
\text { adequada cobertura de } \\
\text { teste sobre riscos de } \\
\text { produtos definidos? }\end{array}$ & $\begin{array}{l}\text { Produto típico de trabalho: Plano de teste } \\
\text { com técnicas de projeto de teste. } \\
\text { Exemplos de técnicas de teste: Partição } \\
\text { de Equivalência; Análise do Valor Li- } \\
\text { mite; Tabela de Decisão; Teste de Tran- } \\
\text { sição de Estados; Teste de Caso de Uso }\end{array}$ \\
\hline $\begin{array}{l}\text { (3) Existem objetivos de } \\
\text { teste definidos com base } \\
\text { nas necessidades e objeti- } \\
\text { vos de negócio? }\end{array}$ & $\begin{array}{l}\text { Produto típico de trabalho: Objetivos } \\
\text { de testes rastreáveis aos objetivos de } \\
\text { negócio. Exemplos de Objetivos de } \\
\text { Teste:Validar a adequação do produto } \\
\text { para uso; Prevenir a ocorrência de de- } \\
\text { feitos em operação; Verificar a conformi- } \\
\text { dade quanto a padrões externos; Forne- } \\
\text { cer visibilidade sobre a qualidade do pro- } \\
\text { duto }\end{array}$ \\
\hline $\begin{array}{l}\text { (4)Foi desenvolvida docu- } \\
\text { mentação para apoiar a } \\
\text { implementação do ambi- } \\
\text { ente de teste? }\end{array}$ & $\begin{array}{l}\text { Produto típico de trabalho: Documento de } \\
\text { apoio a implementação do ambiente de } \\
\text { teste. Exemplos: Guia para instalação } \\
\text { do ambiente; Guia de operação do ambi- } \\
\text { ente; Guia de manutenção do ambiente }\end{array}$ \\
\hline $\begin{array}{l}\text { (5) Os objetivos de teste } \\
\text { são revisados periodica- } \\
\text { mente? }\end{array}$ & $\begin{array}{l}\text { Produto típico de trabalho: Revisão dos } \\
\text { objetivos de teste. Exemplo de revisão: } \\
\text { Reunião com os interessados para revisar } \\
\text { e discutir a necessidade de mudanças nos } \\
\text { objetivos de teste (registrar em ata) }\end{array}$ \\
\hline
\end{tabular}

\subsection{Estabelecimento da Dependência entre as Questões do Questionário}

Uma pergunta $X$ é considerada dependente de $Y$ quando só é possível obter uma resposta positiva para $X$ se previamente foi atribuída uma resposta positiva para $Y$. Um exemplo que representaria essa situação seria atribuir $X=$ " $O$ projeto de teste foi monitorado ao longo de seu ciclo de vida, comparando o planejado e o realizado em relação aos riscos?" e Y="Foram identificados os riscos do projeto de teste e cada risco foi analisado quanto a sua probabilidade de ocorrência, impacto e prioridade?". Como pode-se observar, não é possível realizar o monitoramento de riscos que não foram previamente definidos.

Esta relação foi construída por meio da avaliação da existência de dependência de cada questão do questionário com relação as demais, gerando uma matriz de dependência entre as questões. A definição desta dependência possui dois objetivos distintos:

1. Detectar inconsistências nas respostas de uma avaliação - utilizar a relação de dependência para verificar inconsistências entre as respostas do questionário, ou seja, se existem respostas "Sim" para questões que dependem de outra questão com resposta "Não"; e

2. Eliminar perguntas dependentes - utilizar a relação de dependência para eliminar as questões que dependem de outras questões com respostas "Não", reduzindo assim o número de questões a serem respondidas.

São disponibilizadas as duas abordagens no arcabouço. Pode-se observar que se a empresa optar por acelerar o tempo da avaliação através da eliminação de questões ela perderá a capacidade de identificar inconsistências. A escolha da abordagem mais adequada pode ser feita de acordo com nível de maturidade da empresa, as empresas mais maduras em processo de teste, por exemplo, podem ficar mais seguras de optar pela eliminação de questões.

\subsection{Suporte Automatizado para Realização da Avaliação}


Foi criada uma planilha eletrônica na qual, para cada questão do questionário definido, é possível visualizar o material de apoio, atribuir uma resposta (Sim, Não, Não se Aplica ou Não Conhecido) e vincular evidências dos artefatos produzidos pela empresa que sustentem as respostas "Sim".

A consolidação do resultado foi baseado no método de avaliação do modelo TMM [4], que também é baseado em questionário. Foi possível utilizar esse método já que as áreas de processo do TMMi são similares as metas do TMM e as metas do TMMi são similares as sub-metas do TMM.

Para a avaliação segundo o TMMi, cada meta de maturidade tem um conjunto de questões associadas. A meta é considerada satisfeita se o número de respostas "Sim" das questões associadas a ela for maior ou igual a $50 \%$, ou seja, se o seu grau de satisfação for médio, alto ou muito alto. A classificação da maturidade a nível de área de processo depende da classificação das metas, uma área de processo é satisfeita se a quantidade de metas satisfeitas associadas a ela for maior ou igual a $50 \%$. O nível é considerado satisfeito se todas as áreas de processos forem consideradas satisfeitas. Esse método foi implementado na planilha eletrônica de maneira que, a partir das respostas atribuídas, o resultado é consolidado automaticamente e é atribuído um nível de maturidade para empresa. Além disso, o suporte automatizado também realiza a eliminação de questões dependentes, considerando a matriz de dependência definida na Seção 3.3.

\section{APLICAÇÃO DO ARCABOUÇO}

$\mathrm{O}$ arcabouço foi aplicado como objetivo de estabelecer o panorama da maturidade das empresas do Estado de Goiás em teste de software e avaliar e obter uma opinião das empresas em relação ao arcabouço desenvolvido. A aplicação foi dividida nas etapas de seleção, aplicação e caracterização socioeconômica, descritas a seguir.

Na etapa de seleção, foi elencado um conjunto de empresas de desenvolvimento de software do Estado de Goiás para aplicação do arcabouço de avaliação. Foram selecionadas empresas com maturidade em processo de desenvolvimento, por acreditar que empresas em que modelos de maturidade de processo já foram introduzidos, por mais que não sejam modelos voltados para o teste de software, poderiam realizar uma avaliação mais efetiva do arcabouço de avaliação. Foram contactadas 18 empresas, 15 concordaram em realizar a aplicação, porém, cinco empresas desistiram da aplicação alegando indisponibilidade de recursos. Entre as 10 empresas que restaram, três são de médio ou grande porte, para permitir a comparação da maturidade de empresas de diferentes portes. Apesar do arcabouço ter sido projetado para o contexto de pequenas empresas, ele considerou integralmente as práticas do modelo TMMi, com isso, não há perdas com sua aplicação em empresas de médio e grande porte.

Na etapa de aplicação, o arcabouço foi aplicado nas empresas selecionadas. Com o objetivo de relatar um panorama realista da maturidade de teste de software das empresas avaliadas, optou-se por realizar a avaliação com base em evidências, ou seja, além de responder o questionário a empresa também realizou o vínculo de evidências para sustentar as respostas "Sim". O vínculo das evidências foi exigido para permitir a realização de uma auditoria visando confrontar as respostas atribuídas com os artefatos gerados pela empresa e resultados esperados pelo modelo para cada questão do questionário. Além disso, a avaliação com base em evidências exige um maior nível de critério por parte do responsável pela aplicação, o que fornece mais parâmetros para realização de uma avaliação mais criteriosa do arcabouço. A metodologia de avaliação com base em evidências é utilizada por modelos de maturidade como CMMi, MPS.Br e TMMi, durante o processo de certificação. Diante disso, foi selecionado em cada empresa um representante com conhecimento no processo de teste da empresa, que aplicou o arcabouço atribuindo respostas para as questões e realizando o vínculo de evidências. O representante da empresa também avaliou e forneceu feedback em relação ao arcabouço. Logo em seguida foi realizado uma visita à empresa para verificar se as respostas atribuídas estavam consistêntes com as práticas da empresa, evidênciadas por meio dos artefatos vinculados a cada questão.

$\mathrm{Na}$ etapa de caracterização socioeconômica, as empresas avaliadas responderam um questionário com questões que objetivavam traçar o perfil das empresas e suas perspectivas em relação a disciplina de teste de software. Foram levantadas informações como idade, porte, tamanho da equipe de teste e qualificação da equipe de desenvolvimento. Essas informações foram utilizadas na consolidação dos resultados apresentada na próxima Seção.

\section{RESULTADOS}

Após a aplicação do arcabouço, os resultados obtidos com sua aplicação foram consolidados. O arcabouço consolida automaticamente os resultados da avaliação com base nas respostas atribuídas para o questionário. Diante disso, depois de confirmada a consistência das respostas, pela auditoria, cada empresa já obteve o resultado do seu nível de maturidade em teste de software. As empresas, por meio de um termo de consentimento, autorizaram a divulgação dos resultados. Para preservar a identidade das empresas que participaram da pesquisa, na apresentação dos resultados, as mesmas serão identificadas por A, B, C, D, E, F, G, H, I e J.

A Tabela 3 mostra, para cada empresa, a sua satisfação aos níveis de maturidade do Modelo TMMi. Conforme pode ser observado, apenas a empresa B conseguiu atingir um dos níveis de maturidade, nível 2, já as demais empresas não atingiram nenhum nível de maturidade. O nível 2 de maturidade indica que a empresa $B$ possui um processo de teste gerenciado e claramente independente da depuração. Nesse nível o teste é planejado, controlado e técnicas de projeto são aplicadas para elaboração de casos de testes. Para atingir o nível 2 foi necessário que a empresa comprovasse, por meio de evidências, a satisfação de todas as áreas de processos relacionadas ao nível, sendo as seguintes: estratégias e políticas de testes, planejamento de teste, controle e monitoramento de teste, execução e projeto de teste e ambiente de teste. Para satisfazer as áreas de processos, a empresa comprovou a satisfação de pelo menos $50 \%$ das metas relacionadas a cada área de processo.

Como pode ser visto na Tabela 3, nove das empresas avaliadas não atingiram nenhum nível de maturidade. Porém, isso não indica necessariamente que a empresa não utiliza nenhuma prática de teste ou não conseguiu atingir nenhuma meta, pode indicar que as práticas utilizadas não foram suficientes para atingir nenhum dos níveis. Algumas dessas 
Tabela 3: Nível de maturidade das empresas avaliadas

\begin{tabular}{|c|c|c|c|c|}
\hline Empresa & Nível 2 & Nível 3 & Nível 4 & Nível 5 \\
\hline A & NS & NS & NS & NS \\
\hline B & S & NS & NS & NS \\
\hline C & NS & NS & NS & NS \\
\hline D & NS & NS & NS & NS \\
\hline E & NS & NS & NS & NS \\
\hline F & NS & NS & NS & NS \\
\hline G & NS & NS & NS & NS \\
\hline H & NS & NS & NS & NS \\
\hline I & NS & NS & NS & NS \\
\hline J & NS & NS & NS & NS \\
\hline
\end{tabular}

empresas, apesar de não atingirem nenhum nível, demostraram realizar algumas das práticas do modelo e conseguiram atingir algumas metas de maturidade. A Tabela 4 mostra o percentual de metas que cada empresa conseguiu atingir. A empresa $\mathrm{B}$, que atingiu o nível 2 de maturidade, foi a empresa que atingiu o maior percentual de metas, $37 \%$. A empresa E não atingiu nenhum nível de maturidade, porém atingiu $30 \%$ das metas. Isso é possível já que as metas que a empresa $\mathrm{E}$ atingiu, não foram relacionadas a apenas um nível de maturidade mas sim distribuídas em vários níveis. As empresas $\mathrm{C}$ e $\mathrm{J}$, atingiram respectivamente $17 \%$ e $25 \%$ das metas. Já as empresas A, D, F, G, H e I, não atingiram nenhuma meta ou um número muito baixo, de no máximo $6 \%$.

Tabela 4: Satisfação de metas.

\begin{tabular}{|c|c|c|c|c|c|c|c|c|c|c|}
\hline Empresa & A & B & C & D & E & F & G & H & I & J \\
\hline Metas (\%) & 0 & 37 & 17 & 0 & 30 & 6 & 1 & 3 & 1 & 25 \\
\hline
\end{tabular}

Apesar de uma das empresas ter se destacado em relação as demais, por ser a única a atingir um nível de maturidade, avaliando a Tabela 4, é possível dividir as empresas em dois grupos. O primeiro, das quatro empresas que possuem mais maturidade em teste de software, por terem atingido um percentual de metas maior ou igual a $17 \%$. O segundo, das seis empresas que possuem menos maturidade em teste de software, por terem atingido um percentual de metas menor ou igual a $6 \%$. Por convenção, de agora em diante, o grupo das empresas A, D, F, G, H e I será chamado de Sem Maturidade e o grupo das empresas B, C, E e J será chamado de Com Maturidade.

Ao consolidar os resultados foi identificado que 26 das 71 metas do TMMi não foram atingidas por nenhuma das empresas avaliadas. Juntas, as 4 empresas Com Maturidade atingiram 43 das 71 metas. Juntas as empresas Sem Maturidade atingiram apenas 7 das 71 metas. Entre as práticas mais adotadas pelas empresas estão planejamento de teste, gerência do ambiente de teste e gerência de incidentes. Entre as práticas que não foram encontradas em nenhuma das empresas avaliadas estão plano de carreira para o teste, avaliação de riscos de produto e medição da qualidade do seu produto.

Os resultados do nível de maturidade das empresas também foram confrontados com o perfil socioeconômico das empresas. A Figura 1 mostra a relação entre a maturidade e a idade das empresas. Pode-se observar que as empresas com mais maturidade são as mais velhas, já que 3 das 4 empresas com maturidade possuem mais de 20 anos e 1 está na faixa entre 11 e 20 anos.

A Figura 2 mostra a relação entre a maturidade e o porte

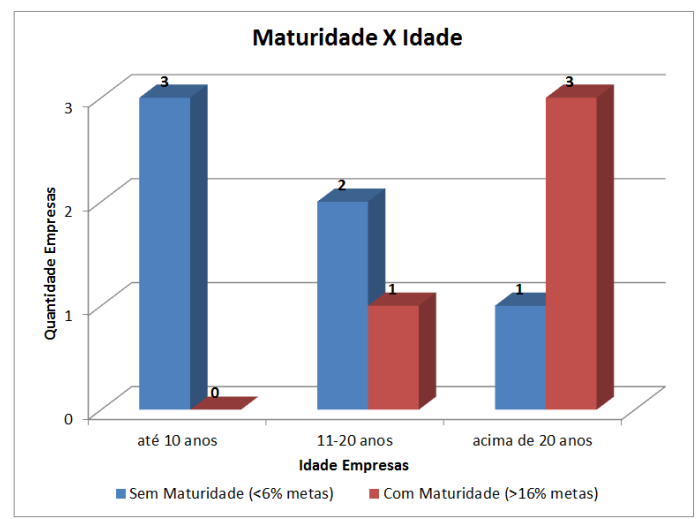

Figura 1: Relação entre maturidade e a idade das empresas avaliadas.

das empresas. As empresas de maior porte possuem mais maturidade, já que 3 empresas com maturidade foram classificadas como médias ou grandes empresas e 1 como empresa de pequeno porte.

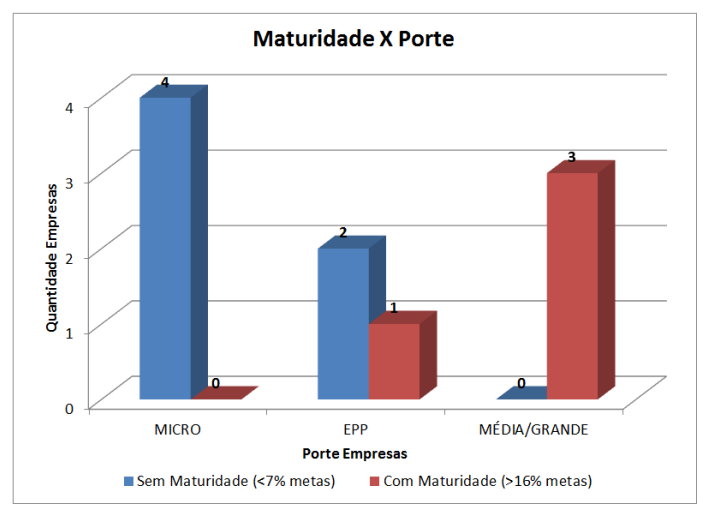

Figura 2: Relação entre maturidade e o porte das empresas avaliadas.

A Figura 3 mostra a relação entre a maturidade e o tamanho da equipe de testes. Não há uma relação direta que indique que quanto maior a equipe de testes maior a maturidade. Apesar de 3 empresas com maturidade possuírem mais de 10 funcionários na equipe de testes, a empresa que atingiu o nível 2 de maturidade possui apenas 3 funcionários na equipe de testes.

A Figura 4 mostra a relação entre a maturidade e as certificações obtidas pelas empresas. Pode-se observar, que as certificações obtidas, mesmo que relacionadas a processo de desenvolvimento apoiaram a maturidade do processo de teste, já que 2 das 4 empresas com maturidade são as únicas que possuem certificações vigentes e as outras 2 já se certificaram, porém a certificação expirou e não foi feita a renovação.

A Figura 5 mostra a relação entre a maturidade e qualificação da equipe de desenvolvimento da empresa. O percentual de qualificação foi calculado utilizando o total de funcionários da área de desenvolvimento com pós-graduação em relação do total de funcionários da área de desenvolvimento. As empresas E e I não são mostradas nesse gráfico 


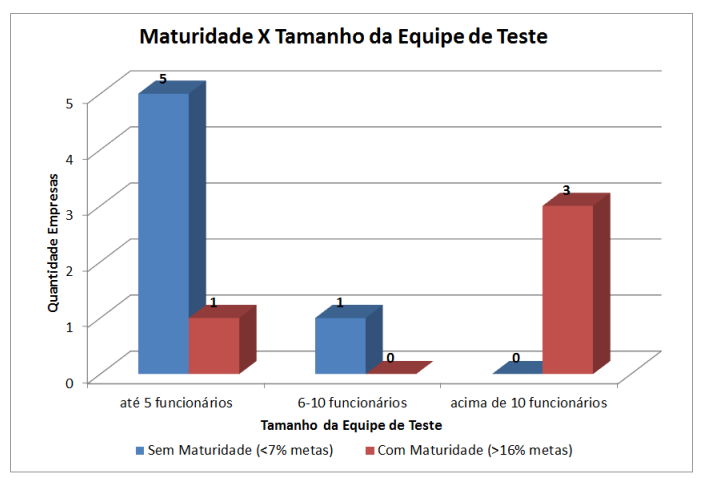

Figura 3: Relação entre maturidade e o tamanho da equipe de teste das empresas avaliadas.

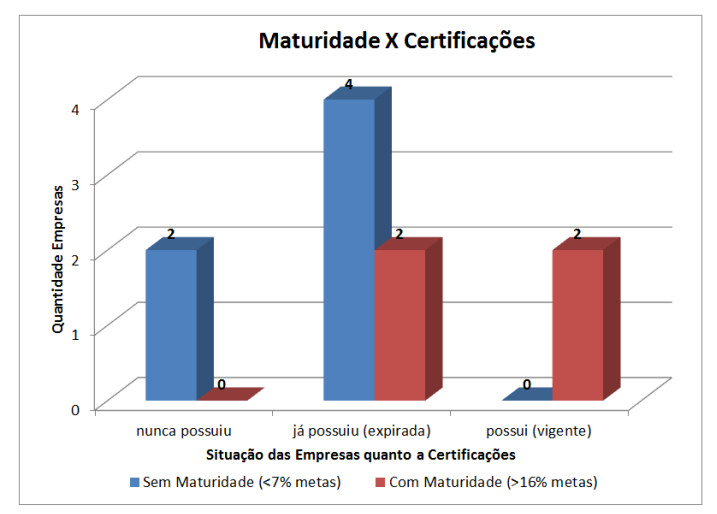

Figura 4: Relação entre maturidade e as certificações obtidas pelas empresas avaliadas.

pois não disponibilizaram essas informações. Pode-se observar que a empresa com maior índice de qualificação, também foi a empresa que obteve maior índice de maturidade. Porém, a empresa $\mathrm{F}$ foi a empresa com o segundo maior índice de qualificação e atingiu apenas $6 \%$ do índice de maturidade.

De maneira geral, podemos perceber com a análise comparativa entre a relação de maturidade e perfil socioeconômico das empresas avaliadas, que em termos quantitativos, a maioria das empresas com maior maturidade, são médias e grandes empresas. Porém, a empresa B, considerada entre todas a empresa mais madura, é uma empresa de pequeno porte, que possui apenas 3 colaboradores envolvidos com as atividades de testes. Isso indica que a maturidade em teste de software não está restrita apenas as grandes empresas, mas também pode ser obtida por micro e pequenas empresas. Para isso, basta que as empresas reconheçam a importância dessa disciplina e busquem a melhoria de seus processos.

Outro resultado que pôde ser consolidado com a aplicação do arcabouço está relacionado com a matriz de dependência disponibilizada no arcabouço. A Tabela 5 mostra o percentual de questões eliminadas com base nas respostas de cada empresa. Pode-se observar um grande ganho em termos de tempo, já que o avaliador não necessitou atribuir respostas para até $65 \%$ das questões, no caso da empresa A. A eliminação de questões é inversamente proporcional ao nível de maturidade das empresas, já que quanto menor o nível de

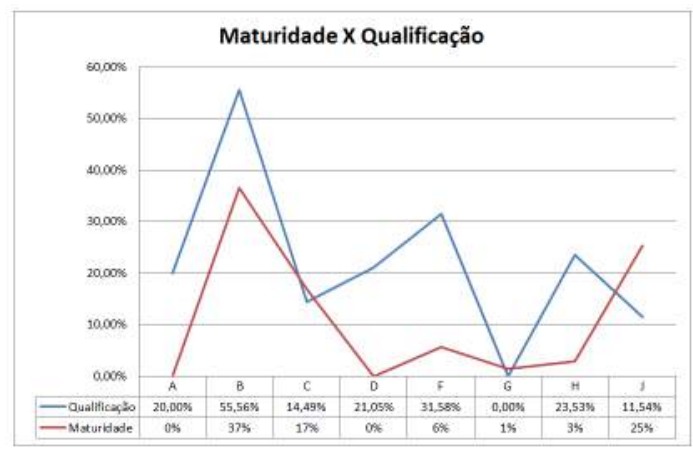

Figura 5: Relação entre maturidade e a qualificação da equipe de desenvolvimento das empresas avaliadas.

maturidade da empresa, mais respostas Não serão atribuídas para as questões e consequentemente, como a eliminação é realizada com base nas respostas iguais a Não, maior será o índice de eliminação de questões.

Tabela 5: Eliminação de questões com base nas respostas e matriz de dependência.

\begin{tabular}{|c|c|c|c|c|c|c|c|c|c|c|}
\hline Empresa & A & B & C & D & E & F & G & H & I & J \\
\hline Questões (\%) & 65 & 26 & 45 & 60 & 33 & 49 & 62 & 37 & 60 & 34 \\
\hline
\end{tabular}

A avaliação do arcabouço foi feita por meio da aplicação do questionário definido na na Tabela 6. Para cada questões, o representante da empresa selecionou uma opção entre as faixas $0-25 \%, 26-50 \%, 51-75 \%$ e $76-100 \%$. A consolidação da avaliação do arcabouço pode ser vista na Tabela 7 . É apresentado para cada questão o percentual de empresas que selecionaram cada faixa de classificação. Por exemplo, $90 \%$ das empresas indicaram que o nível de importância dos exemplos para o vínculo de evidências às questões, questão 4, é entre $76-100 \%$. Também foi apresentado o percentual geral por faixa de classificação, por exemplo, em $68 \%$ das questões, foi selecionada a faixa entre $76-100 \%$. Como pode ser visto, as questões 4 e 3 , respectivamente, foram as questões que obtiveram o maior índice de avaliação, $90 \%$ e $80 \%$ das empresas indicaram a maior faixa de classificação $76-100 \%$. Para as questões 1 e 2, 60\% das empresas selecionaram a maior faixa de classificação. A questão 5, obteve o menor índice de avaliação, tendo a maior faixa de classificação indicada por $50 \%$ das empresas. De maneira geral o arcabouço foi avaliado positivamente pelas empresas, já que $90 \%$ das empresas indicaram as faixas de classificação entre $51-100 \%$ e nenhuma das empresas indicou a faixa $0-25 \%$.

Tabela 6: Questões utilizadas para avaliar o arcabouço.

\begin{tabular}{|c|l|}
\hline Seq. & Questão \\
\hline 1 & Qual o nível de clareza das perguntas do questionário? \\
\hline 2 & Qual o nível de clareza dos exemplos fornecidos? \\
\hline 3 & $\begin{array}{l}\text { Qual o nível de importância dos exemplos para a compre- } \\
\text { ensão das perguntas? }\end{array}$ \\
\hline 4 & $\begin{array}{l}\text { Qual o nível de importância dos exemplos para o vínculo de } \\
\text { evidências às questões? }\end{array}$ \\
\hline 5 & $\begin{array}{l}\text { Qual o nível de alinhamento do vocabulário utilizado nas } \\
\text { questões e exemplos à sua realidade? }\end{array}$ \\
\hline
\end{tabular}

Considerando o cenário de aplicação e os resultados obtidos foram identificadas as algumas ameaças a validade desta 
Tabela 7: Consolidação da avaliação do questionário.

\begin{tabular}{|c|c|c|c|c|c|c|}
\hline Classificação & $\mathbf{1}$ & $\mathbf{2}$ & $\mathbf{3}$ & $\mathbf{4}$ & $\mathbf{5}$ & Geral \\
\hline $0-25 \%$ & $0,0 \%$ & $0,0 \%$ & $0,0 \%$ & $0,0 \%$ & $0,0 \%$ & $0,0 \%$ \\
\hline $26-50 \%$ & $10,0 \%$ & $10,0 \%$ & $10,0 \%$ & $10,0 \%$ & $10,0 \%$ & $10,0 \%$ \\
\hline $51-75 \%$ & $30,0 \%$ & $30,0 \%$ & $10,0 \%$ & $0,0 \%$ & $40,0 \%$ & $22,0 \%$ \\
\hline $76-100 \%$ & $60,0 \%$ & $60,0 \%$ & $80,0 \%$ & $90,0 \%$ & $50,0 \%$ & $68,0 \%$ \\
\hline
\end{tabular}

pesquisa: (a) a aplicação do arcabouço foi realizada com base em uma avaliação pessoal e subjetiva dos artefatos gerados pelo processo da empresa e as questões e exemplos disponibilizados pelo arcabouço. Diante disso, o responsável pela aplicação, pode não ter entendido as questões ou exemplos, deixando de vincular evidências praticadas na empresa; (b) o arcabouço foi aplicado em 10 empresas do Estado de Goiás, sendo 7 MPEs. Não é conhecido se a maturidade em teste de software apontada neste trabalho é a realidade da maioria das MPEs de software do Estado de Goiás, do Brasil e/ou do mundo. Entretanto, com base nos resultados apresentados e no conhecimento da realidade de várias outras empresas do Estado, que não participaram dessa pesquisa, acredita-se que esse resultado representa a realidade das demais empresas do Estado; e (c) o arcabouço não foi aplicado em empresas certificadas no Modelo TMMi, essa aplicação poderia verificar se os resultados apresentados pelo arcabouço são similares aos resultados obtidos com uma avaliação oficial realizada por consultores credenciados pelo modelo.

\section{CONCLUSÃO}

Neste artigo foi mostrada a importância das MPEs de desenvolvimento de software para economia mundial e que essas empresas necessitam melhorar continuamente a qualidade dos seus produtos para se adequarem as exigências do mercado. Dada a importância do Teste de Software para produção de software com maior qualidade foi criado um arcabouço para avaliação do nível de maturidade em Teste de Software segundo o modelo TMMi, adequado a realidade das MPEs.

Com os resultados da aplicação do arcabouço constatou-se que o questionário objetivo, o material de apoio relacionado a cada questão e a planilha que consolida automaticamente o resultado da avaliação permitem que MPEs, mesmo com baixo nível de maturidade, realizem uma autoavaliação da sua maturidade em Teste de Software. As empresas avaliaram positivamente o arcabouço ao apontarem o nível de adequação do arcabouço, $68 \%$ dos itens avaliados foram classificados entre $76-100 \%$, maior faixa disponibilizada e $90 \%$ dos itens foram classificados entre $51-100 \%$.

Com a aplicação do arcabouço em dez empresas do Estado de Goiás, foi constatado que o nível de maturidade das empresas do estado é baixa. Apenas uma das empresas avaliadas atingiu um nível de maturidade do modelo, nível 2. Além disso, a maturidade das empresas foi confrontada com suas informações socioeconômicas. A empresa com maior nível de maturidade, empresa B, é uma empresa de pequeno porte, com apenas 3 colaboradores que executam atividades de teste, porém, é a empresa que possui a equipe de desenvolvimento e teste mais qualificada e possui certificação vigente em um modelo de maturidade de desenvolvimento de software. As microempresas não possuem nenhuma maturidade. Já as médias e grandes empresas, apesar de não atingirem nenhum nível de maturidade, executam algumas das práticas do modelo e conseguiram atingir algumas metas.

Apesar da baixa maturidade apontada pela avaliação de maturidade realizada, as empresas reconhecem a importância da disciplina de teste de software e pretendem realizar investimentos nessa área. O que justifica o trabalho desenvolvido, que está sendo utilizado por algumas das empresas avaliadas, como referência para realização de melhorias no seu processo de teste.

\section{REFERÊNCIAS}

[1] M. Q. A. A., S. Ramachandram, and A. M. A. An evolutionary software product development process for small and medium enterprises (SMEs). pages 298-303. IEEE, Oct. 2008.

[2] A. F. Araujo, C. L. Rodrigues, A. M. R. Vincenzi, C. G. Camilo-Junior, and A. F. Silva. A Framework for Maturity Assessment in Software Testing for Small and Medium-Sized Enterprises. pages 225-230, 2013.

[3] J. Brodman and D. Johnson. What small businesses and small organizations say about the CMM. pages 331-340. IEEE Comput. Soc. Press, Aug. 2002.

[4] I. Burnstein. Practical Software Testing: a process-oriented approach. Springer-Verlag New York, Inc., Secaucus, NJ, USA, 2002.

[5] A. Gentile. Associação brasileira das empresas de software - mercado brasileiro de software - panorama e tendências. Technical report, 2014.

[6] E. Höhn. KITeste - A Framework of Knowledge and Test Process Improvement. $\mathrm{PhD}$ thesis, USP, São Carlos - SP - Brazil, June 2011. (in Portuguese).

[7] M. Khokhar, K. Zeshan, and J. Aamir. Literature review on the software process improvement factors in the small organizations. pages $592-598$. IEEE Comput. Soc. Press, May 2010.

[8] C. Laporte, S. Alexandre, and A. Renault. Developing international standards for very small enterprises. Computer, 41(3):98-101, Mar. 2008.

[9] M. A. Montoni, A. R. Rocha, and K. C. Weber. MPS.BR: a successful program for software process improvement in brazil. Softw. Process, 14(5):289-300, Sept. 2009.

[10] S. Ng, T. Murnane, K. Reed, D. Grant, and T. Chen. A preliminary survey on software testing practices in australia. pages 116-125. IEEE, 2004.

[11] SEI. Capability maturity model integration - CMMI version 1.3 .

[12] Softex. Melhoria do processo de teste brasileiro MPT.Br versão 3.0.

[13] Softex. Evaluations published MPS.BR, Mar. 2012. (in Portuguese).

[14] SQI. Appraisal assistant, 2007.

[15] T. Tayamanon, T. Suwannasart, N. Wongchingchai, and A. Methawachananont. TMM appraisal assistant tool. pages 329-333. IEEE, Aug. 2011.

[16] TMMi-Foundation. Test maturity model integration TMMi version 3.1.

[17] T. Varkoi, T. Makinen, and H. Jaakkola. Process improvement priorities in small software companies. page 555. Portland Int. Conf. Manage. Eng. \& Technol. PICMET, Aug. 2002. 